\title{
Bisphenol A
}

National Cancer Institute

\section{Source}

National Cancer Institute. BisphenolA. NCI Thesaurus. Code C152071.

A diphenylmethane derivative with two hydroxyphenyl groups. Bisphenol A (BPA) is a

colorless solid that is used in the synthesis of commercial plastics, including

polycarbonates and epoxy resins, which are incorporated into a wide variety of consumer goods. Ingested BPA may exhibit estrogenic effects. Exposure to BPA may increase the risk of certain cancers. 